Jean Noël MARIEN ${ }^{1}$ Guillaume DeLaunaY ${ }^{2}$

${ }^{1}$ Cirad

URP Bsef

Biens et services des écosystèmes forestiers tropicaux :

l'enjeu du changement global

TA C-105 / D Campus international de Baillarguet

34398 Montpellier Cedex 5

France

${ }^{2}$ Starlight Products SA 129, chemin de Croisset BP 4026

76021 Rouen Cedex 3 France

\title{
La tara, Caesalpinia spinosa : espèce agroforestière emblématique des vallées interandines au Pérou
}

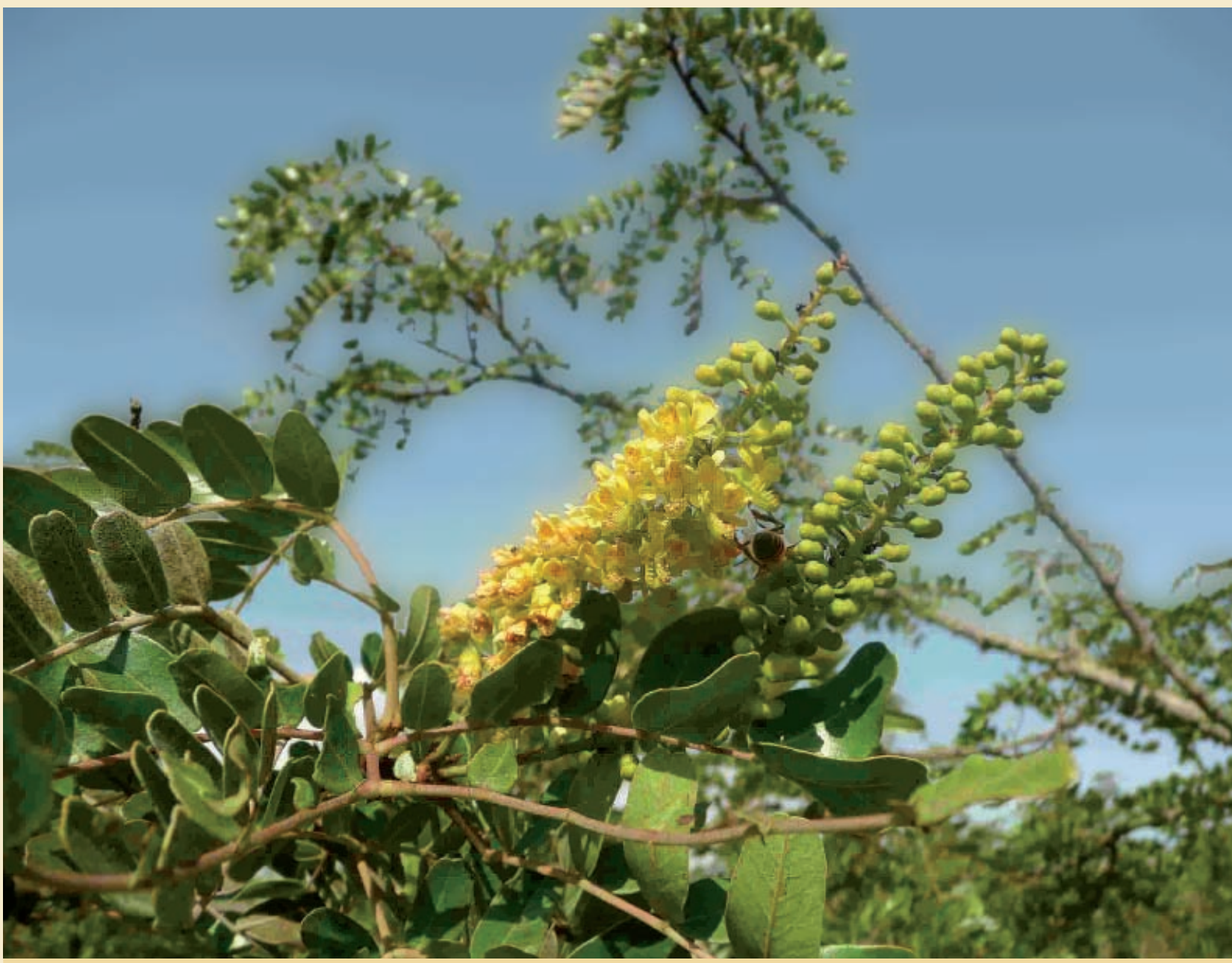

Floraison de Caesalpinia spinosa, Mol. Photo J.-N. Marien. 


\section{RÉSUMÉ}

\section{LA TARA, CAESALPINIA SPINOSA : ESPĖCE AGROFORESTIÈRE EMBLÉMATIQUE DES VALLÉES INTERANDINES AU PÉROU}

La tara Caesalpinia spinosa Mol., espèce multi-usage traditionnellement utilisée par les populations rurales au Pérou, fait l'objet depuis une dizaine d'années d'une attention particulière pour ses gousses, riches en tanins, et ses graines valorisées comme gomme dans l'industrie alimentaire. Cette espèce est présente dans plusieurs pays andins, mais son aire principale se situe au Pérou. La typologie de la ressource naturelle montre que l'essentiel des surfaces et de la production est constitué par différents systèmes agroforestiers (haies vives, cultures associées, arbres isolés...). Les forêts naturelles sont des reliques le plus souvent en mélange avec d'autres essences. Enfin, les plantations (paysannes ou industrielles) ne représentent encore qu'une très faible partie des surfaces ; mais cela évolue assez rapidement, compte tenu des prix actuels des gousses, très attractifs pour les producteurs. La filière est très organisée, mais pour l'essentiel informelle. Elle est composée de trois types d'acteurs. Les producteurs (ou récolteurs) sont les familles de paysans au sein de communautés rurales pauvres. Les acheteurs sont de plusieurs niveaux en fonction de leur positionnement vis-à-vis des vendeurs et de leur capacité de financement. La filière tara revêt une importance économique majeure, qu'il s'agisse de productions issues de peuplements naturels (ou subspontanés) ou de plantations (à petite ou grande échelle). Son développement peut aussi être analysé en termes de création de biens et services écosystémiques variés dans le contexte des changements globaux (climatiques et autres).

Mots-clés : Caesalpinia spinosa, tara, tanins, gomme, ressource naturelle, agroforesterie, filière de production, biens et services écosystémiques, Pérou.

\section{ABSTRACT}

\section{THE TARA TREE, CAESALPINIA SPINOSA: AN AGROFORESTRY SPECIES, EMBLEM OF PERU'S ANDEAN VALLEYS}

Tara tree, Caesalpinia spinosa (tara) is traditionally used for a variety of purposes by rural populations in Peru, and for about 10 years has been the focus of particular attention for its tannin-rich pods and seeds used for gum in the food industry. The species occurs in several Andean countries, but principally in Peru. The typology of this natural resource shows that growth and production occurs essentially in different agroforestry systems (live hedges, intercropping, isolated trees, etc.). In the wild, it is mainly found as relict forest mixed with other species. Finally, plantations (peasant farming or industrial) account for only a very small proportion of the total area, although this is changing quite rapidly in view of current very attractive prices paid to producers for the pods. Although the sector is highly organised, it is still essentially informal. It operates on three levels. Producers (or gatherers) are peasant families in poor rural communities. Buyers operate on several levels depending on their relations with vendors and their financing capacity. The tara crop is of considerable economic importance, whether produced from natural (or subspontaneous) forest or from plantations (small or large scale). Its development can also be analysed in terms of the creation of different ecosystem goods and services in the context of global change (climate change and others).

Keywords: Caesalpinia spinosa, tara, tannins, gum, natural resource, agroforestry, production sector, ecosystem goods and services, Peru.

\section{RESUMEN}

LA TARA, CAESALPINIA SPINOSA: ESPECIE AGROFORESTAL EMBLEMÁTICA DE LOS VALLES INTERANDINOS DE PERÚ.
La tara, Caesalpinia spinosa Mol., especie multipropósito tradicionalmente utilizada por las poblaciones rurales en Perú, lleva unos diez años siendo objeto de especial atención por sus vainas, ricas en taninos, y sus semillas, valorizadas como goma en la industria alimentaria. Esta especie está presente en varios países andinos, pero su área principal se sitúa en Perú. La tipología de este recurso natural muestra que la mayoría de las áreas de crecimiento y producción están constituidas por diferentes sistemas agroforestales (cercas vivas, cultivos asociados, árboles aislados,...). Los bosques naturales son formaciones relictas en las que suele haber mezclas con otras especies. Por último, las plantaciones (campesinas o industriales) sólo representan, por ahora, una parte muy pequeña de la superficie ocupada, aunque esta situación está evolucionando con rapidez debido a que los precios actuales de las vainas son muy atractivos para los productores. El sector está muy organizado, pero generalmente de forma informal. Se compone de tres niveles: los productores (o cosechadores), que son las familias de campesinos de comunidades rurales pobres. Los compradores, estructurados en varios niveles según su posicionamiento y capacidad de financiamiento frente a los vendedores. El sector de la tara tiene una importancia económica transcendental, tanto en producciones procedentes de masas naturales (o subespontáneas) como en plantaciones (de pequeño o gran tamaño). Su desarrollo también puede analizarse en términos de creación de bienes y servicios ecosistémicos variados en un contexto de cambios globales (climáticos y otros).

Palabras clave: Caesalpinia spinosa, tara, taninos, goma, recurso natural, agroforestería, sector de producción, bienes y servicios ecosistémicos, Perú. 


\section{Introduction}

Connue, domestiquée, utilisée depuis des temps immémoriaux et considérée comme "l'or vert des Incas ", la tara, Caesalpinia spinosa Mol., fait depuis plusieurs années l'objet d'un intérêt renouvelé pour la valeur de ses produits, gomme alimentaire issue de l'endosperme et tanins pour l'industrie issus de la gousse.

Une étude a été menée en 2008, en partenariat entre le Cirad ${ }^{1}$ et l'entreprise Starlight Products, pour mieux appréhender la réalité de cette ressource et l'organisation de sa filière. Cet article présente les principaux résultats obtenus dans la description de la ressource naturelle et de la filière de production.

La littérature sur la tara n'est pas très abondante. Ce n'est que depuis une dizaine d'années que cette espèce multi-usage, traditionnellement utilisée par les populations rurales, fait l'objet d'une attention particulière pour ses gousses, riches en tanins, et ses graines, valorisées comme gomme dans l'industrie alimentaire.

\section{Une ressource variée pour des usages multiples}

Caesalpinia spinosa est endémique du Pérou, mais aussi marginalement de plusieurs pays de la zone andine (Bolivie, Chili...). Elle occupe la zone des formations végétales de type "matorral arbustivo" (sec et subhumide) dans les vallées et les coteaux interandins. Cette espèce se trouve dans une gamme large de sols et de conditions climatiques. S'agissant d'une espèce très plastique, l'aire naturelle est diffuse ; mais il n'existe pas de carte de distribution de l'espèce. Selon les informations de l'Inrena ${ }^{2}$, son aire de prédilection se situe sur les versants atlantiques (800 à $2800 \mathrm{~m}$ ) et pacifiques (1 600 à $2800 \mathrm{~m}$ ).

La présence de Caesalpinia spinosa est étroitement associée à l'homme et occupe une place spécifique dans l'organisation du territoire des vallées et coteaux interandins (INRENA, 2007 ; FAO ${ }^{3}, 1998$; VILLANUEVA, 2007).

Les peuplements naturels (tableau I) s'étendent le plus souvent sur des surfaces de quelques ares à quelques hectares. Ils occupent des zones refuges ou délaissées par les autres occupations de l'espace. Il s'agit principalement de taillis ou taillis sous futaies âgées et aux cimes assez peu développées. Ils sont souvent en mélange avec d'autres espèces arbustives telles que Schinus molle (molle) ou diverses espèces d'acacias. Ces peuplements sont utilisés pour le bois de feu et le pâturage

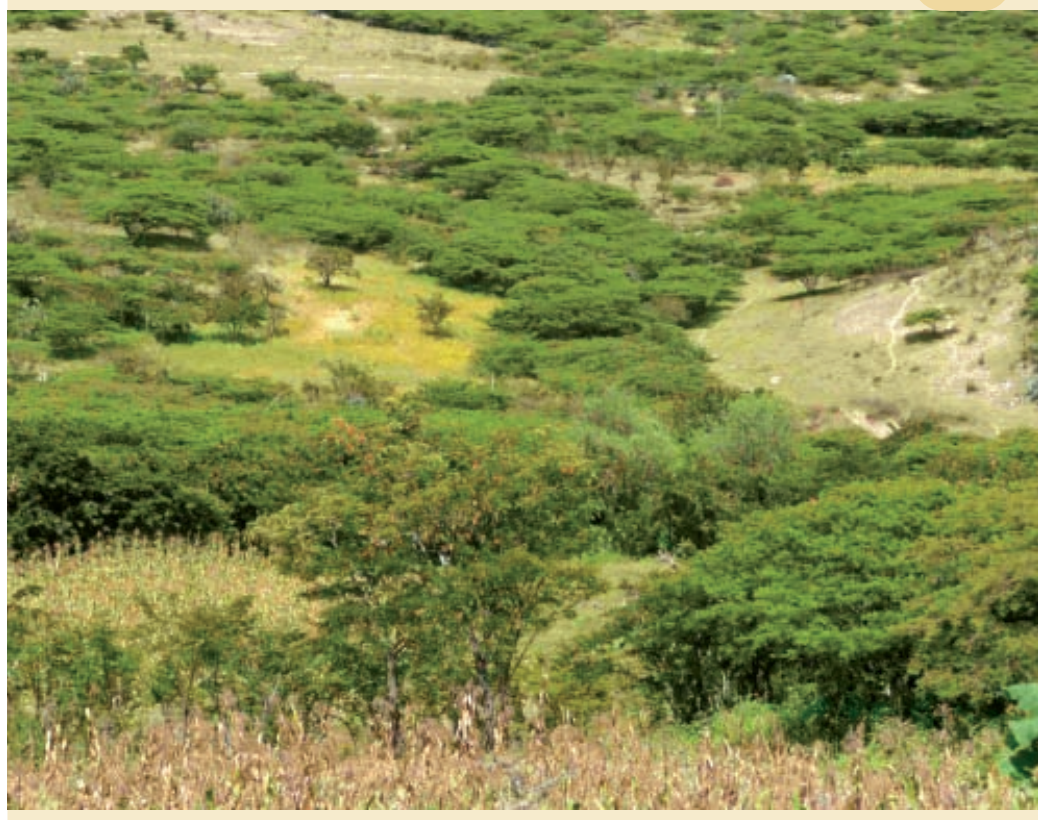

Peuplement relique naturel de tara, en mélange avec Acacia sp. et Shinus sp. Photo J.-N. Marien. extensif. La productivité de ces peuplements est assez faible, à la fois à cause du développement aérien, mais aussi en raison de la présence de nombreux parasites et maladies.

La tara est domestiquée depuis de nombreux siècles et l'essentiel de sa distribution géographique est étroitement lié aux activités humaines. Elle est tellement intégrée dans le paysage que la plupart des auteurs considèrent à tort ces systèmes agroforestiers comme des forêts naturelles. C'est un très bon exemple d'espèce multi-usage (bois énergie, outillage agricole, charpentes, fruits, fourrage, clôtures). Elle est introduite sous forme de jeunes plants le long des pistes, champs, prairies ou jardins et constitue le plus souvent des haies vives. Elle est également souvent plantée en intercalaire avec d'autres spéculations agricoles telles que les figuiers de barbarie, Opuntia ficus indica, pour la culture des cochenilles. Enfin, il est possible de la trouver sous forme d'arbres isolés dans des champs (céréales). Les surfaces des parcelles agricoles sont assez réduites (souvent moins de $1 \mathrm{ha}$ ) et pourraient ainsi s'apparenter au système de bocage.

Le développement de la partie aérienne de la tara, donc sa productivité en gousses, est directement lié aux pratiques culturales locales et aussi à l'âge des arbres, les haies étant souvent assez âgées.
Tableau I.

Synthèse des informations bibliographiques sur la répartition des surfaces et les productions observées.

Boisement naturel

Agroforesterie

30

Proportion des surfaces (\%)

Production moyenne
Plantation

65
${ }^{1}$ Centre de coopération internationale en recherche agronomique pour le

développement.

${ }^{2}$ Instituto Nacional de Recursos Naturales.

${ }^{3}$ Food and Agriculture Organization of the United Nations. 


\section{Un environnement socio- économique complexe}

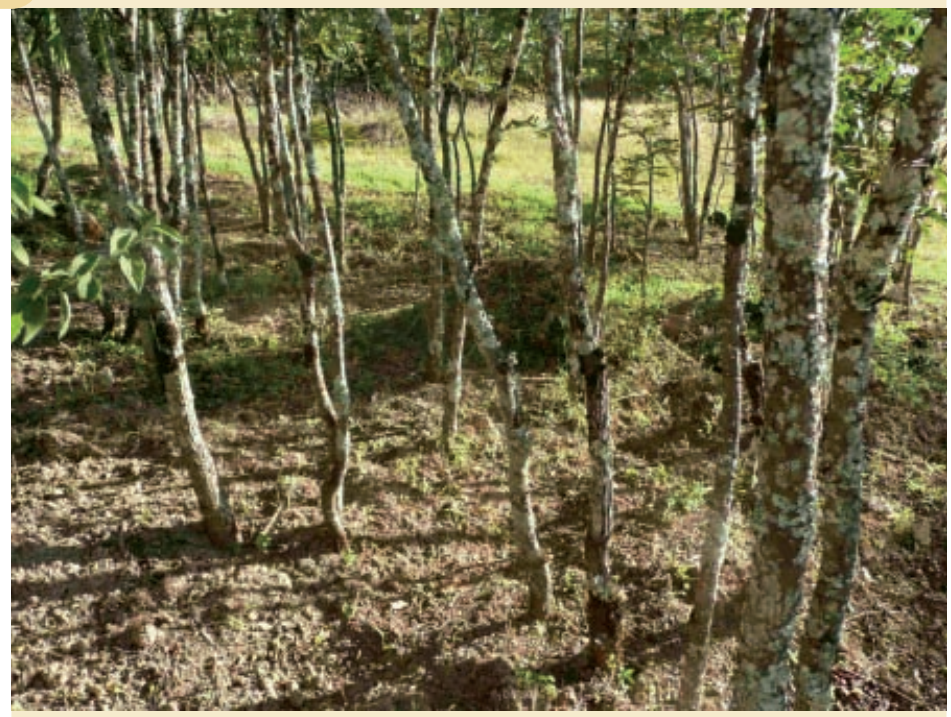

Aspect des cépées en peuplement naturels. Photo J.-N. Marien.

Depuis une quinzaine d'années, la tara rencontre un intérêt croissant et les premiers projets significatifs de plantations ont vu le jour pendant les années 1990. Contrairement aux systèmes agroforestiers, elles visent spécifiquement la production de gousses et occupent $100 \%$ des terrains mis en culture. Elles s'apparentent donc directement à la mise en place de vergers fruitiers. Depuis le début des années 2000 et avec l'augmentation de la demande, l'attrait pour les plantations a augmenté considérablement. La productivité des plantations, quelle que soit leur origine, leur taille ou leur mode de financement, est étroitement liée à la mise en œuvre de bonnes pratiques culturales (VIGO , QUIROZ, 2007 ; QUISPE ARBILDO, 2007).

Les plantations paysannes sont de taille réduite $(0,1$ à 5 ha). Elles sont gérées directement par le propriétaire de la parcelle. Leur développement et les productivités attendues sont étroitement liés au savoir-faire et au degré d'intervention. Parmi les plantations visitées, seule une petite partie respecte les règles de bonne gestion. On constate souvent des défauts (emplacement, entretien, taille...) qui retardent significativement le développement de l'arbre et sa mise à fruits.

Les plantations industrielles apparaissent depuis peu. Les plus anciennes datent du début des années 2000. Plusieurs projets sont en cours de réalisation ou en attente de financement. Ces plantations, réalisées sur des surfaces significatives (supérieures à 5 ha), sont caractérisées par l'utilisation de techniques intensives (travail du sol, irrigation, fertilisation, taille...) avec des investissements importants. Le manque de disponibilité en terres de bonne qualité est un frein important à l'extension de ces plantations.

Il est difficile de parler de surfaces car la plupart des peuplements se rattachent à la catégorie agroforestière et sont majoritairement constitués d'arbres isolés ou de haies. Néanmoins, les chiffres cités ci-dessous ne paraissent pas incompatibles avec les observations effectuées au cours des visites. La proportion de bosquets naturels est sans doute inférieure à $30 \%$ car la majorité des peuplements sont plus proches des systèmes agroforestiers (figure 1).
Les zones rurales du Pérou sont très habitées et les voies de communication (y compris les pistes muletières) nombreuses. La tara est dans sa grande majorité incluse dans un système agroforestier, à proximité immédiate des champs et cultures, la rendant très accessible pour la récolte. Le caractère familial de la récolte permet de collecter des arbres isolés ou des petits bosquets sans efforts particuliers. Le niveau de prix actuel, incitatif, provoque un intérêt fort et une collecte importante. Seuls les bosquets naturels sont en général libres d'accès (propriété d’État), mais ils sont peu productifs et ne représentent approximativement que $15 \%$ des surfaces répertoriées en tara.

La tenure foncière est assez stricte. Le sol est attribué soit à des petits propriétaires, soit à des collectivités (communautés locales). La réforme foncière socialiste a éliminé les grandes propriétés et attribué les terres à des ayants droit locaux, malheureusement le plus souvent ni préparés ni éduqués. Il n'existe pas, dans les zones considérées des vallées et coteaux interandins, de terrains vacants sur lesquels on pourrait trouver une ressource non attribuée.

Le vol de gousses semble un fléau assez répandu. Les acheteurs achètent sans rechercher l'origine des produits et le prix de vente actuel peut inciter des personnes non détentrices de droits sur des arbres à voler les gousses. Des chiffres pouvant aller jusqu'à 50 \% de la production commercialisée sont cités. De plus, le vol se fait souvent sur des fruits pas encore mûrs et contribue ainsi à diminuer la valeur technologique des produits. Difficiles à maîtriser à petite échelle, les risques de pillage sont encore plus importants pour les plantations industrielles et devront être intégrés dans les modalités de gestion.

Rapidement, des projets de développement ont été initiés par des organisations non gouvernementales locales ou des organismes gouvernementaux (Idesi ${ }^{4}$, Fondempleo, Solid Perú, Agroservis, A.C. Tierra, Adefor ${ }^{5}$...) sur cofinancements internationaux $\left(\mathrm{Gtz}^{6}, \mathrm{Snv}^{7}\right.$...). Les actions ont porté sur l'appui technique, la fourniture de plants, la formation, le microcrédit,

${ }^{4}$ Instituto de Desarollo del Sector Informal.

${ }^{5}$ Asociacion civil para la investigacion y el desarollo forestal.

${ }^{6}$ Deutsche Gesellschaft für Technische Zusammenarbeit.

Nederland Development Organization.

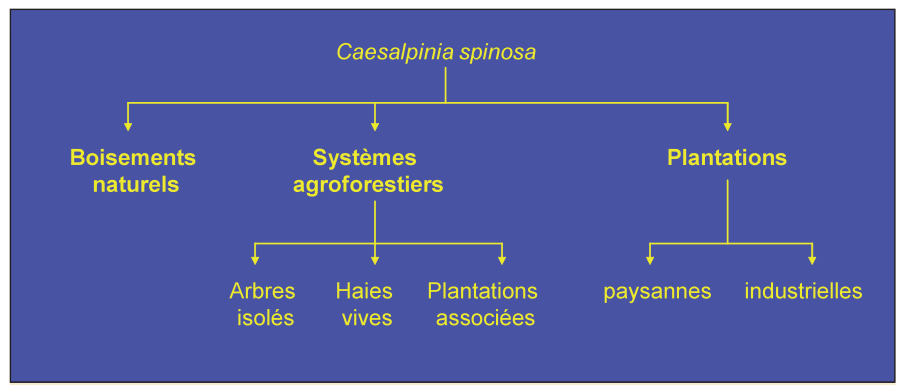

Figure 1.

Répartition des formations végétales et de la production de gousses de tara. 


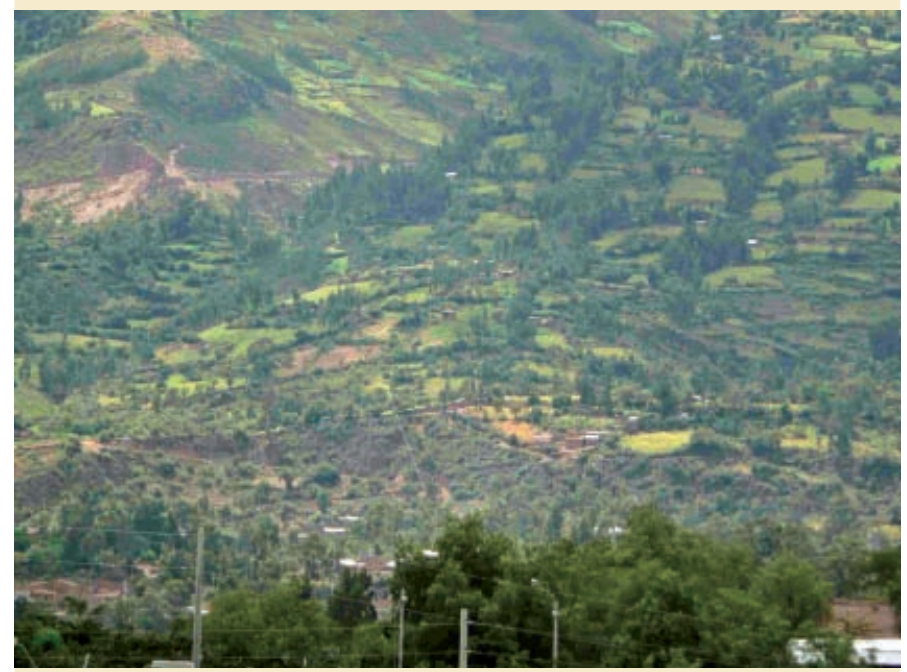

Paysage très répandu de bocage (Huanta, Ayacucho) en bas de pente, avec cultures entourées de haies de tara. Noter les bosquets d'eucalyptus (bois de feu et de service) et la densité de l'habitat. Photo J.-N. Marien.

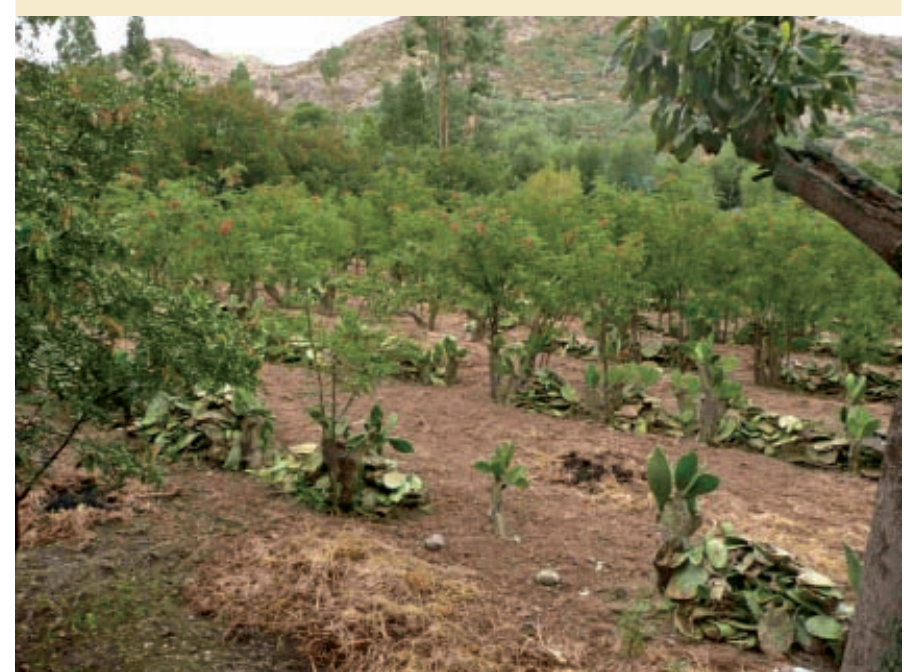

Agroforesterie avec interlignes pour la cochenille d'Opuntia sp.

Photo J.-N. Marien.

mais aussi sur l'organisation de coopératives de producteurs et l'insertion des producteurs dans le circuit de commercialisation. Cet environnement, destiné à fournir emplois ruraux et revenus alternatifs aux populations défavorisées, a encouragé le développement de plantations paysannes et une gestion plus intensive des systèmes agroforestiers. Plusieurs milliers d'hectares sont concernés par ces pratiques. Certains organismes (Snv) souhaitent développer des partenariats entre les sociétés de négoce et les producteurs (ANONYmE, 2004).

Les graves troubles politiques et la violence qui ont régné sur une partie du Pérou au cours de la décennie 1980 ont gravement atteint les zones de production, et en particulier la région d'Ayacucho. Les coupes de bois pour l'énergie domestique et le chauffage ont détruit une partie significative de la ressource, laquelle se reconstitue progressivement, compte tenu de l'intérêt économique suscité par la tara.

\section{Une filière organisée, mais largement informelle}

Une des approches pour estimer la production consiste à analyser les flux de récolte commercialisée. En effet, l'administration délivre des permis pour toute transaction et les chiffres avancés sont généralement considérés comme fiables par les acteurs de la filière. Pour l'ensemble du pays, l'Inrena a estimé que la production nationale de gousses de tara est passée de 2,5 millions de tonnes en 2000 à plus de 26 millions de tonnes en 2006 (compte tenu du taux de conversion de $65 \%$ entre la gousse et la poudre), soit une production largement décuplée en sept campagnes de récolte.

Trois régions (Cajamarca, La Libertad et Ayacucho) totalisent, en 2006, plus des deux tiers du total récolté et les marges de progression de la récolte sont impressionnantes puisque la production totale est passée de 8297 tonnes en 2004 à 22486 tonnes en 2005 et 26035 tonnes en 2006. Cette progression est surtout le fait des provinces de Cajamarca et d'Ayacucho, où l'organisation de la récolte a été dynamisée par la mise en place de projets de développement sur cofinancements internationaux (Gtz, Snv) et la présence d'organismes nationaux d'appui (Idesi). Certaines régions ont vu leur niveau de récolte stagner, voire régresser ( $\mathrm{La} \mathrm{Li}$ bertad, Lambayeque) (NovoA, RAMIREZ, 2007).

Plus de $80 \%$ de la production provient des systèmes agroforestiers. La part des peuplements naturels est faible $(12 \%)$. Celle des plantations est encore minime, mais leur contribution à la récolte totale devrait augmenter assez rapidement, au fur et à mesure de la montée en puissance des projets, tant paysans qu'industriels.

La filière s'organise suivant trois niveaux distincts (INCAgro, 2004 ; AvENDANO TORRES, 2008).

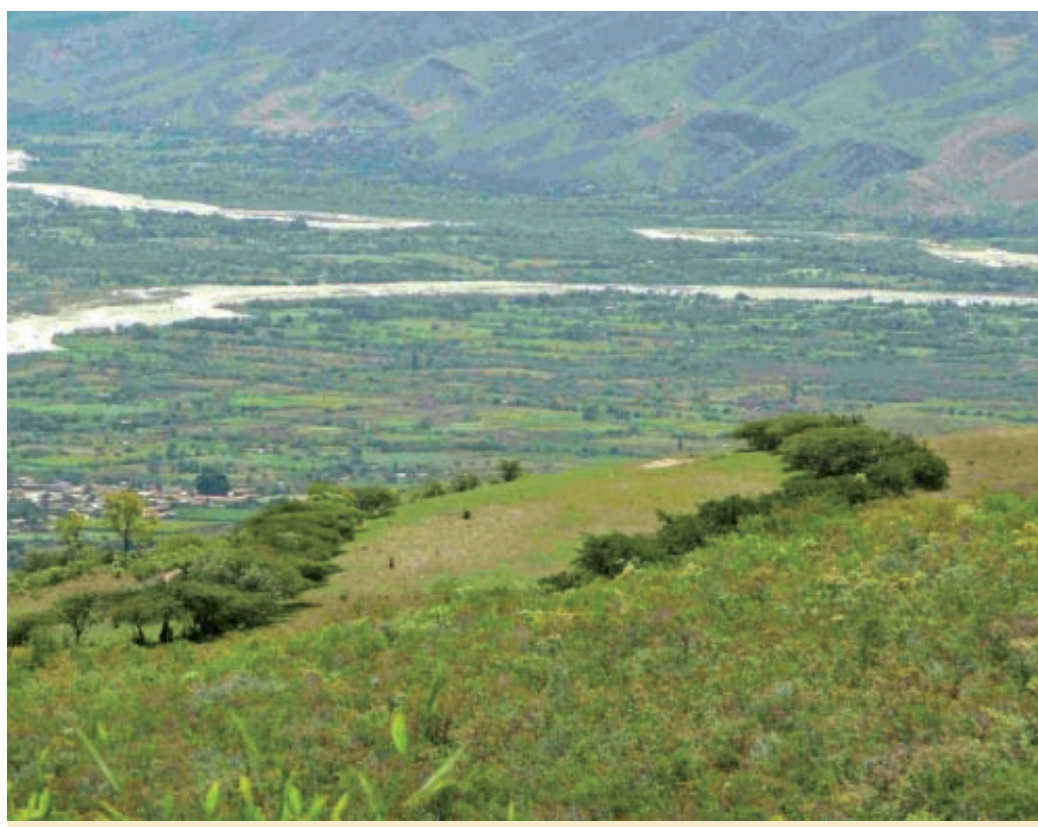

Haie vive en bord de champ (planté en tara). Photo J.-N. Marien. 


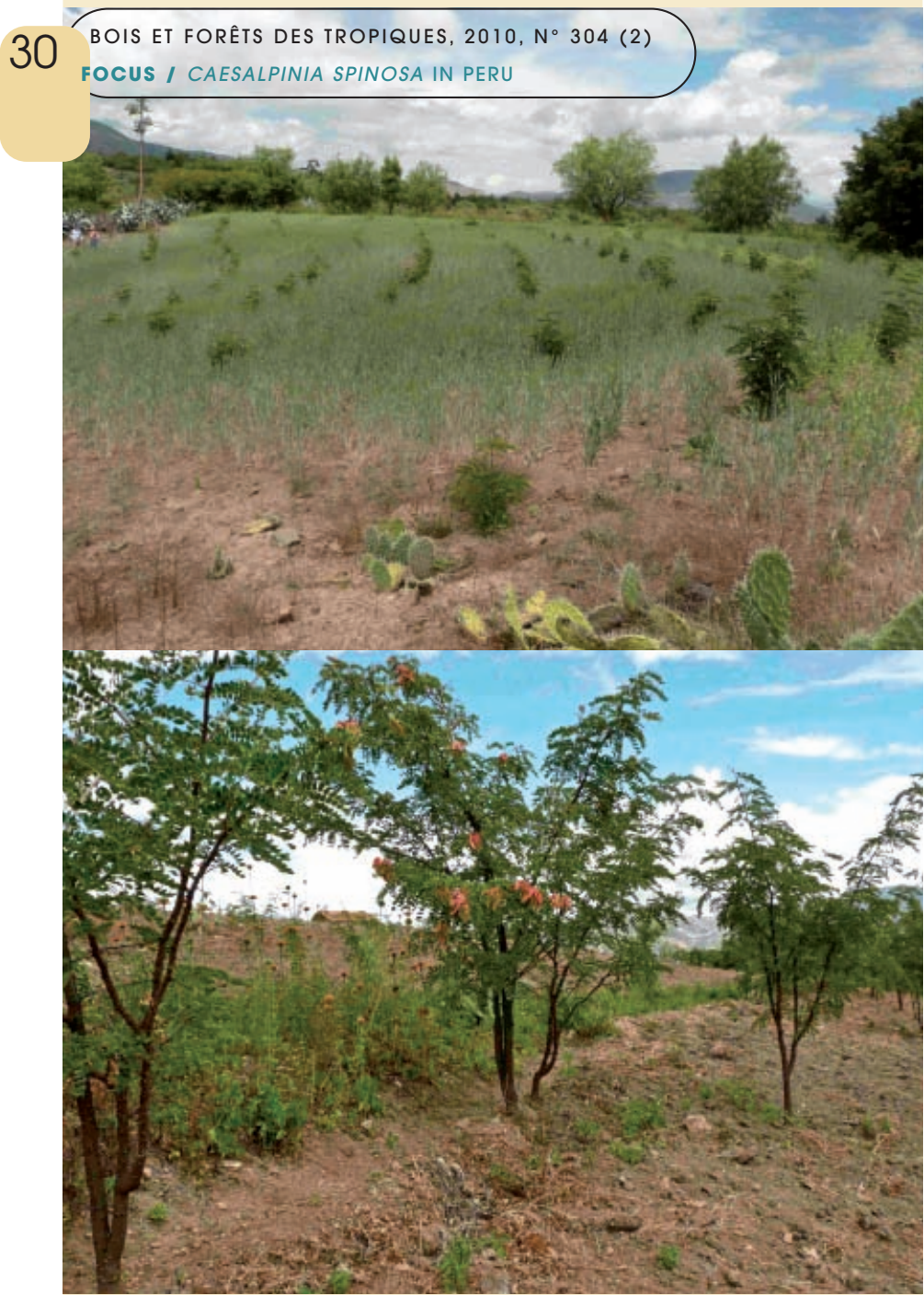

Plantation paysanne réussie (Yuraccracay, Ayacucho), six mois avec culture intercalaire et trois ans avec irrigation par rigoles et entretiens. Photo J.-N. Marien.

\section{Les producteurs}

Ils sont atomisés. Chaque producteur possède des droits (formels ou traditionnels)) sur une quantité d'arbres faible, en moyenne de l'ordre de 50 arbres, soit moins d'une tonne par producteur. L'immense majorité de ces producteurs ne fait aucun investissement, se contentant de récolter les gousses, le plus souvent en famille. Les gousses sont mises à sécher, puis placées dans des sacs et transportées jusqu'au premier point de vente. Ils ne sont ni formés, ni encadrés. Leur seul intérêt est guidé par le prix de vente et ils n'ont pas conscience de l'importance de la qualité, ce qui entraîne souvent des récoltes de gousses immatures ou des tentatives de fraude (mélange, poids, vol...). La vente de la tara apporte un complément ponctuel de revenu.

Certains producteurs (quelques pourcents) investissent dans des plantations avec fertilisation et irrigation (plantations paysannes). D'autres sont en relation avec les organisations locales de développement, qui leur procurent un appui technique et organisationnel. Dans ces cas, la productivité est plus forte et le contrôle de la qualité plus affirmé, mais les investissements sont plus élevés.

\section{Les acheteurs-collecteurs (acopiadores)}

Ils constituent le deuxième maillon de la chaîne. Les gousses de tara sont transportées, le plus souvent par mulet ou véhicule à moteur, jusqu'au point de vente le plus proche. Il s'agit souvent des marchés hebdomadaires qui ont lieu dans la plupart des villes et villages. Il peut aussi s'agir des échoppes fixes situées en ville ou à proximité. C'est à ce niveau de la filière que se font les demandes d'autorisation et les déclarations obligatoires de ressource pour l'administration.

\section{Plusieurs types d'intermédiaires}

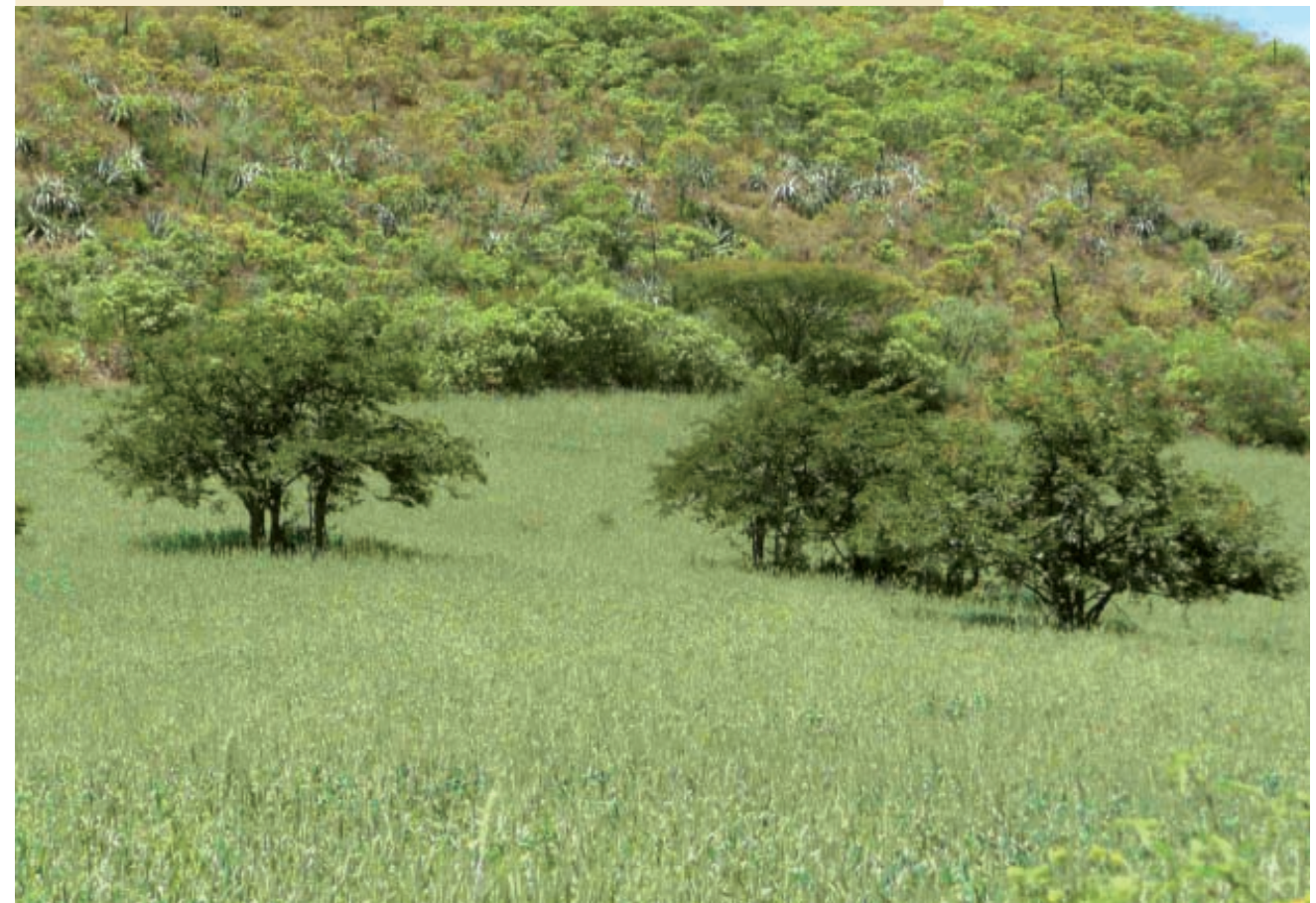

Les acheteurs primaires se tiennent au débouché des pistes d'accès aux marchés ruraux. Si certains producteurs sont fidélisés, la plupart comparent les prix de vente avant de céder leur marchandise au mieux-disant. II y a donc à ce niveau une véritable concurrence et surenchère. Le paiement se fait au comptant, après pesage et contrôle du contenu des sacs. Le contrôle ne porte pas sur la qualité des gousses et ces acheteurs ne sont pas irréprochables.

Les acheteurs secondaires ont pignon sur rue. Ils disposent le plus souvent d'un local commercial où ils stockent des produits achetés directement aux producteurs, mais aussi parfois aux acheteurs primaires. Ils com-

Arbres isolés dans les champs.

Photo J.-N. Marien. 


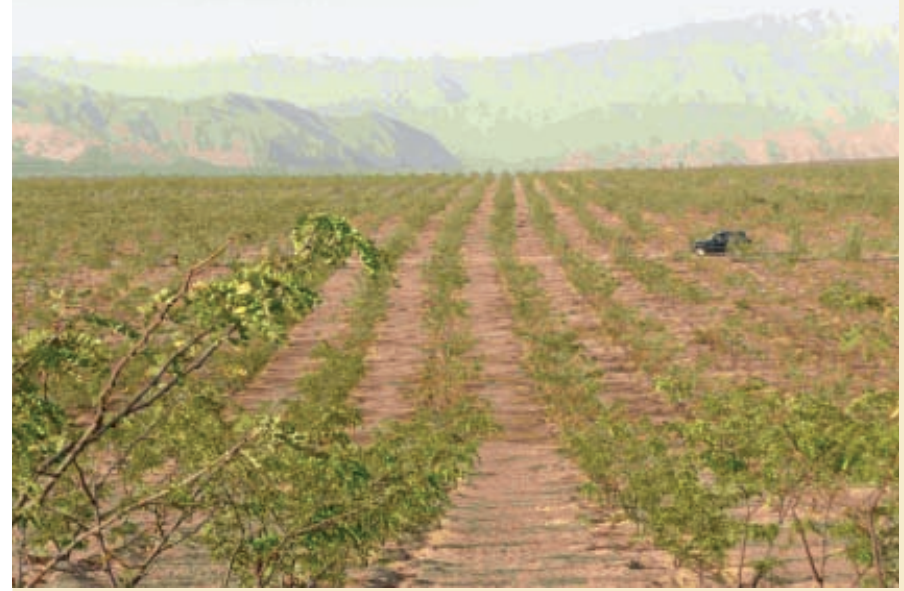

Plantation industrielle (2 ans).

Photo J.-N. Marien.

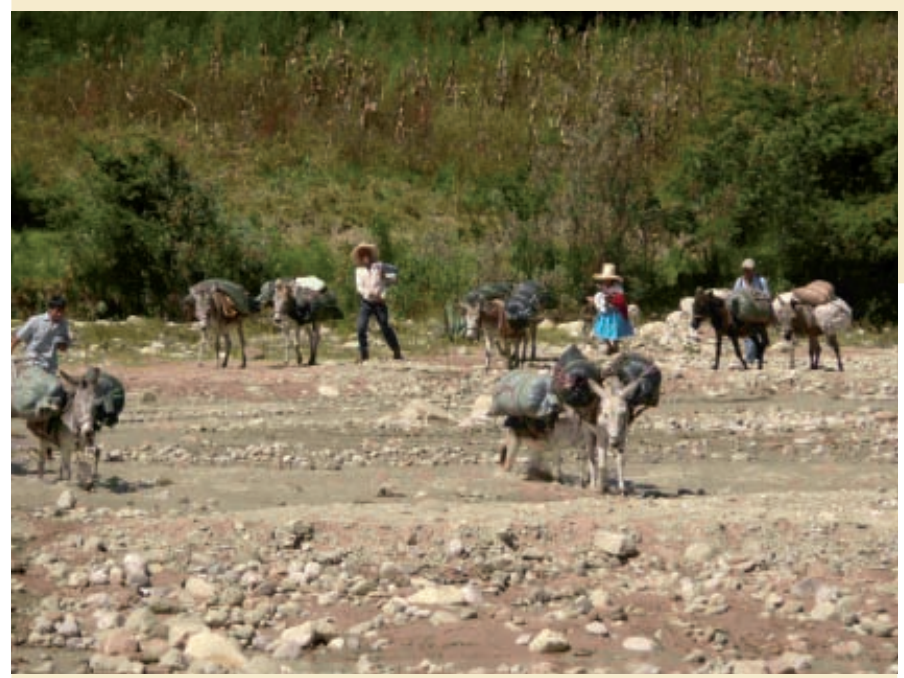

Producteurs/récolteurs se rendant au marché de San Marcos (Cajamarca).

Photo J.-N. Marien.

mercialisent également d'autres produits (cochenilles...). À ce niveau aussi, la qualité n'est pas prise en compte, ou alors à la marge. Les acheteurs principaux sont localisés dans les villes. Ils achètent les produits aux acheteurs primaires, et surtout secondaires. Ces acheteurs travaillent souvent pour une société de transformation, mais ils peuvent aussi vendre au plus offrant. C'est le premier niveau auquel la société de transformation peut se référer pour tenter d'obtenir un produit de qualité et homogène.

Les sociétés de transformation sont pour la plupart basées à Lima et prospectent sur l'ensemble du pays, à l'exception de deux d'entre elles installées dans les capitales provinciales et avec un rayon d'approvisionnement régional. Depuis quelques années, une concentration forte du secteur s'opère et actuellement moins d'une dizaine de sociétés se partagent le marché. La concurrence est forte. Les volumes mobilisés par ces sociétés dépendent des prix d'achat qu'elles proposent et de leur capacité à mobiliser les acheteurs au travers d'un réseau de négociateurs quadrillant le territoire. La présence effective des agents (directs ou indirects) de ces sociétés est un facteur important de visibilité et de crédibilité vis-à-vis des acheteurs. Enfin, la capacité des sociétés à payer au comptant, voire à faire des avances de trésorerie aux acheteurs, est un élément fort de motivation.

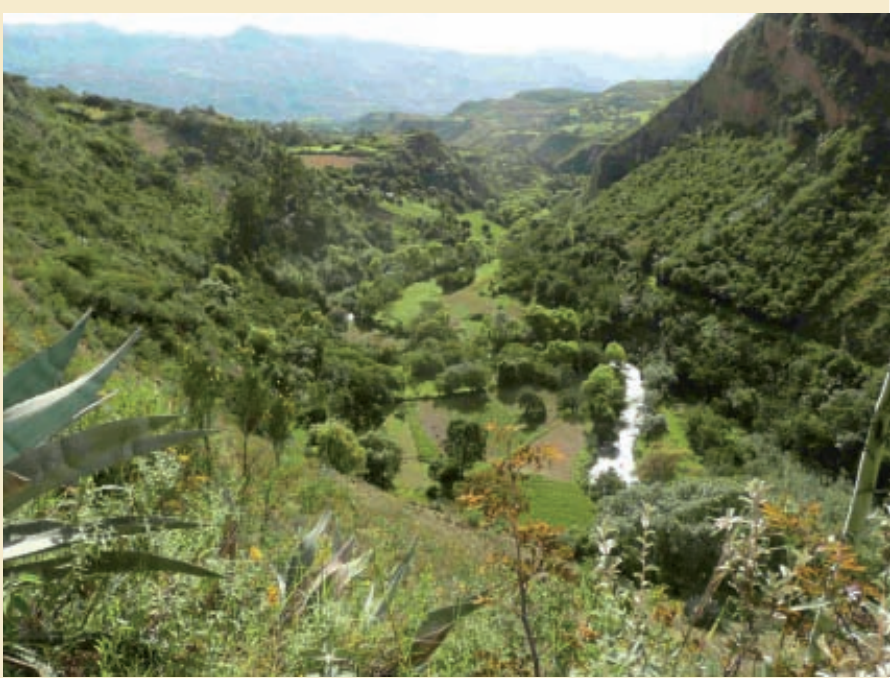

Fond de vallée avec haies de tara et bosquets naturels en bas de pente (Cajabamba, Cajamarca). Photo J.-N. Marien.

\section{Une fourniture importante de biens et services écosystémiques}

La tara, espèce locale des vallées interandines, dispose d'une capacité d'adaptation significative, puisqu'on la retrouve, sous des modalités diverses, dans des contextes plus difficiles (voire extrêmes, avec par exemple les plantations sur dunes côtières en climat désertique), ou dans d'autres pays, voisins ou non. La contribution de la filière au bilan économique a été déjà évoquée, en incluant les différents aspects des combinaisons agroforestières (agriculture, élevage...), sachant que cette filière est un véritable outil de création de revenus et de réduction de la pauvreté dans des zones rurales isolées et déshéritées.

En outre, l'espèce fournit de nombreux biens et services écosystémiques importants à plusieurs niveaux d'agrégation territoriale.

À l'échelle de la parcelle, cette légumineuse, par sa capacité à fixer l'azote atmosphérique, permet tout à la fois une meilleure croissance des espèces associées et de ne pas recourir aux engrais azotés, chers et dont le coût énergétique (et donc en carbone) est très élevé. Cette capacité lui permet de se développer dans des zones arides, sur des sols très pauvres (sableux et salés).

À l'échelle du bassin versant, la tara est un véritable marqueur du territoire et donc de la propriété et de la sécurisation foncières. Plantée en haies ou en bosquets, elle contribue à la lutte contre l'érosion, sur des versants souvent très pentus. La fourniture de biomasse (bois et autres produits) diminue d'autant la pression sur les ressources forestières naturelles, souvent largement dégradées. Dans les zones très arides, elle entraîne la création de nouveaux écosystèmes boisés dont le rôle protecteur ou restaurateur n'est plus à démontrer.

À l'échelle du pays, la tara, essence à croissance rapide, contribue à l'amélioration du bilan carbone et devrait pouvoir être éligible aux mécanismes de financement $M$ dp $^{8}$ et Redd ${ }^{9}$.

\footnotetext{
${ }^{8}$ Mécanisme de développement propre.

${ }^{9}$ Reduced emissions for forest degradation and deforestation.
} 


\section{Conclusion}

La tara, Caesalpinia spinosa, est un exemple méconnu d'association agroforestière traditionnelle au Pérou. L'essentiel de la ressource est en effet cultivé en association avec des cultures vivrières (fruits et légumes), agricoles (céréales) ou des produits à forte valeur ajoutée (cochenille).

Le développement de nouveaux usages et l'industrialisation des procédés de transformation ont permis le développement d'une filière nationale et internationale dynamique et compétitive. Après des décennies d'oubli et de dégradation de la ressource, l'intérêt pour cette espèce est à l'origine d'un renouveau de sa gestion et de la création d'une ressource plantée, donc mieux contrôlée et appropriée par les acteurs de la filière. Fait suffisamment rare pour être noté, les producteurs sont les principaux bénéficiaires, au moins à court terme et dans un environnement social et humain difficile et souvent conflictuel, d'une situation où la demande excède l'offre. Le développement de la collecte et d'une ressource issue de plantations devrait cependant contribuer à terme à professionnaliser et équilibrer la filière.

L'évolution récente des plantations est forte du côté des petits producteurs dans un contexte agro-écologique natif (la " selva » originelle entre 800 et $2800 \mathrm{~m}$ ) et moins rapide dans des régions de néo-implantation avec irrigation et culture intensive. Il est possible d'estimer qu'il existe aujourd'hui environ une trentaine d'initiatives qui pourraient totaliser plus de 2000 hectares et sont susceptibles de doubler à court terme la production totale actuelle.

Acheteur secondaire à Huanta (Ayacucho).

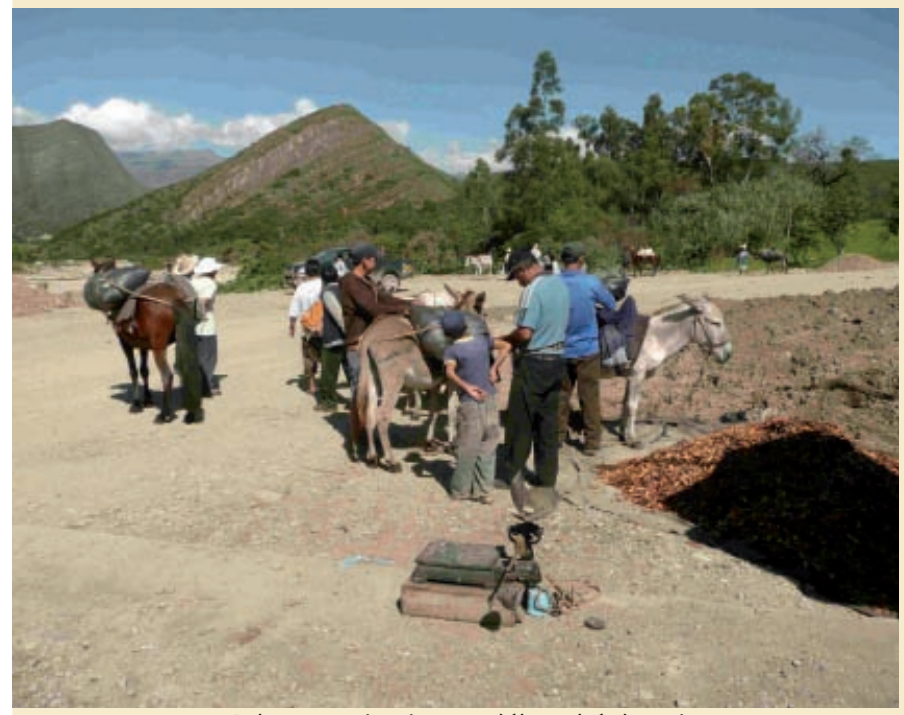

Acheteur primaire, au débouché des pistes

(San Marcos).

Photo J.-N. Marien.

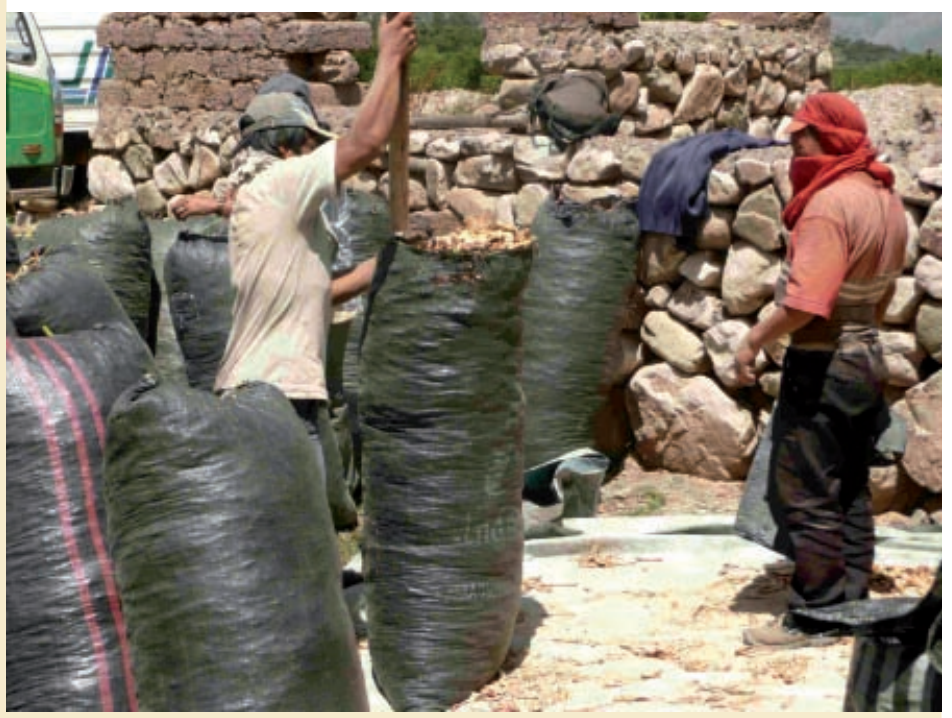

Acheteur primaire, sur le marché de La Grama (Cajamarca). Photo J.-N. Marien. 


\section{Références bibliographiques}

ANONYME, 2004. Proyecto de introducción y mejoramiento de la produccion de tara en los districtos de Tipan y Viraco. Informe final, $10 \mathrm{p}$.

AVENDANO TORRES E., 2008. Conociendo la cadena productiva de la tara en Ayacucho. Solid Perú, Snv, rapport, 104 p.

FAO, 1998. Especies arboreas y arbustivas para las zonas aridas y semiaridas de America Latina. Caesalpinia spinosa (Mol.). Synthèse sur cette espèce et sur sa culture, $12 \mathrm{p}$.

INCAGRO, 2004. Document de projet pour la promotion de la chaîne de production de la tara à Ayacucho. Trujillo, Pérou, Instituto de Desarrollo del Sector Informal (Idesi).

INRENA, 2007. Importancia del recurso Tara o Taya. Lima, Pérou, Inrena, brochure de vulgarisation, $6 \mathrm{p}$.

INRENA, 2008. Statistiques de production et d'exportation. Lima, Pérou, Inrena.

MARQUINA R., 2007. El aprovechamiento de la tara (Caesalpinia spinosa Molina) en la comunidad campesina San Pedro de Pampay, Ayacucho, considerando el enfoque de género. Lima, Pérou, InfoAndina, 22 p.

VIGO E., QUIROZ V., 2007. El cultivo de la tara en Cajamarca. Guide pratique de culture de la tara. Gtz, A.C. Tierra, 39 p.

NOVOA S., RAMIREZ E. K., 2007. Evaluación del estado de conservación de Caesalpinia spinosa (tara) en el departamento de Ayacucho. Lima, Pérou, Inrena, Serie de publicaciones de flora y fauna silvestres, $9 \mathrm{p}$.
QUISPE ARBILDO H., 2007. Proyecto de pre-factibilidad para la instalación de 100 hectáreas de Tara (Caesalpinia spinosa) en Jayanca - Lambayeque, Péru. 26 p.

VILLANUEVA C. M., 2007. La tara : « El oro verde de los Incas ». Lima, Pérou, Ediciones Agrum, Universidad Nacional Agraria La Molina, 164 p.

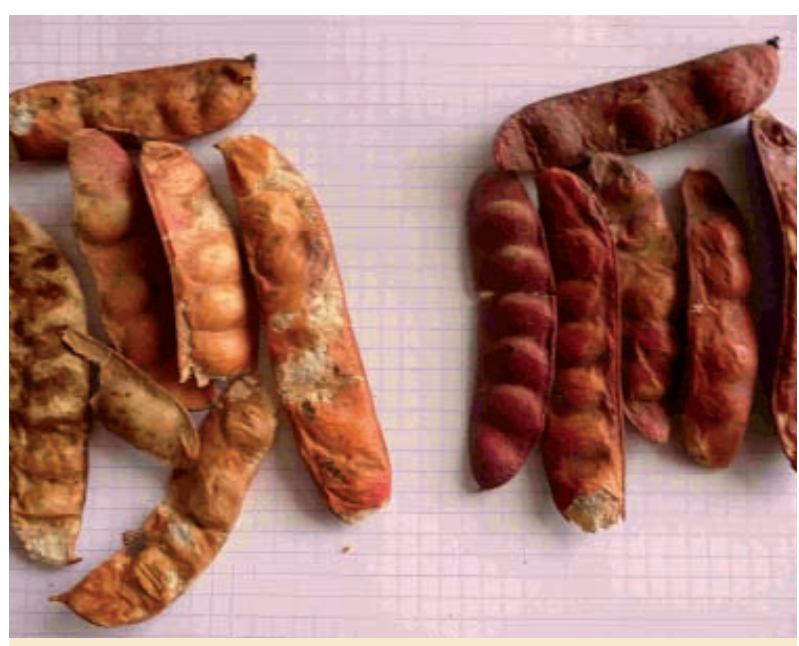

Gousses de tara. Photo J.-N. Marien.

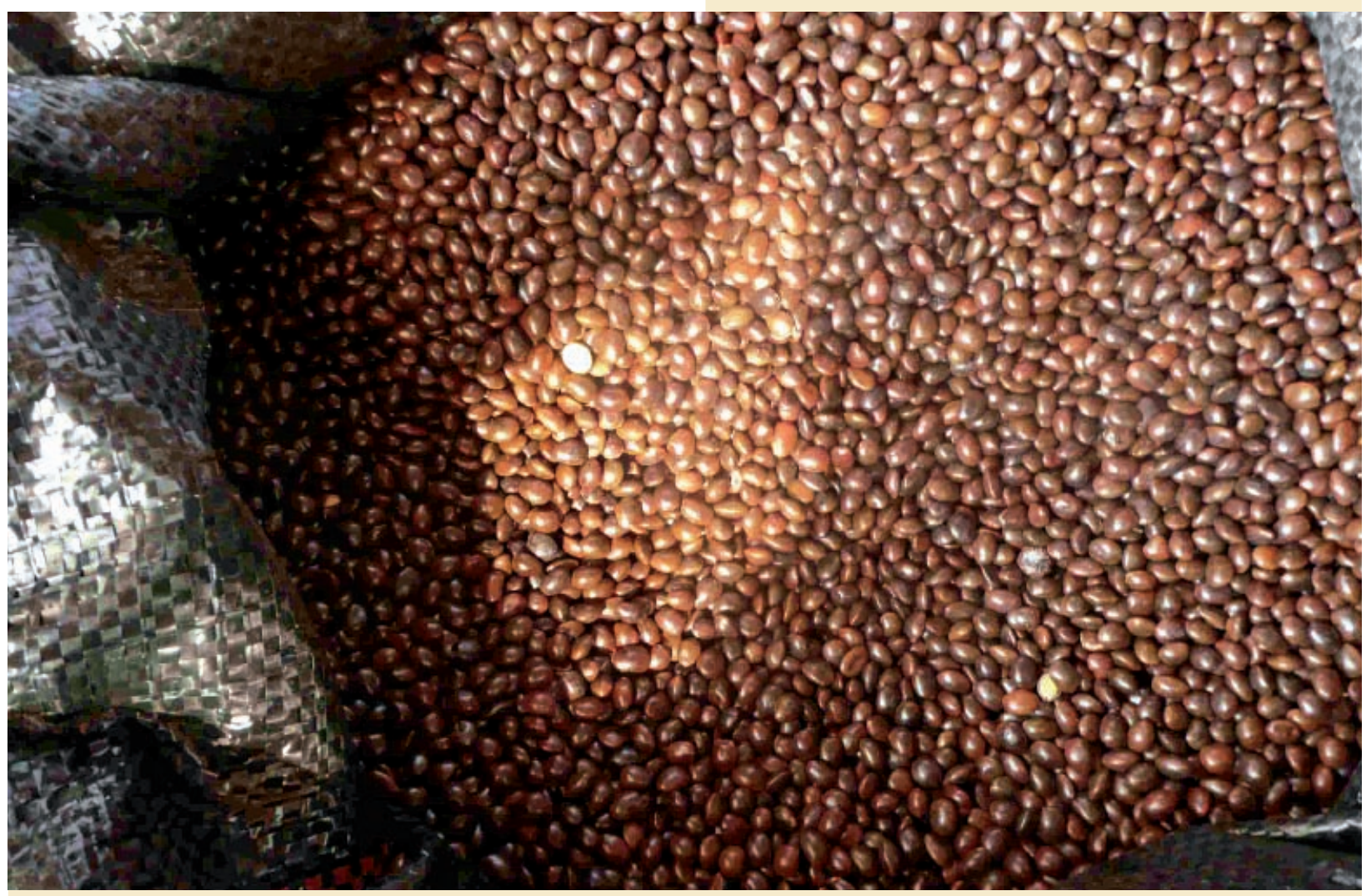

Graines de tara.

Photo J.-N. Marien. 